\title{
Decaying Dark Atom Constituents and Cosmic Positron Excess
}

\author{
K. Belotsky, ${ }^{1,2}$ M. Khlopov, ${ }^{1,2,3}$ C. Kouvaris, ${ }^{4}$ and M. Laletin ${ }^{1}$ \\ ${ }^{1}$ National Research Nuclear University "Moscow Engineering Physics Institute”, Moscow 115409, Russia \\ ${ }^{2}$ Centre for Cosmoparticle Physics "Cosmion", Moscow 115409, Russia \\ ${ }^{3}$ APC laboratory 10, rue Alice Domon et Léonie Duquet, 75205 Paris Cedex 13, France \\ ${ }^{4}$ CP $P^{3}$-Origins, University of Southern Denmark, Campusvej 55, 5230 Odense, Denmark
}

Correspondence should be addressed to M. Laletin; mnlaletin@mail.ru

Received 30 November 2013; Revised 3 March 2014; Accepted 20 March 2014; Published 16 April 2014

Academic Editor: Jean-René Cudell

Copyright ( $92014 \mathrm{~K}$. Belotsky et al. This is an open access article distributed under the Creative Commons Attribution License, which permits unrestricted use, distribution, and reproduction in any medium, provided the original work is properly cited. The publication of this article was funded by SCOAP $^{3}$.

\begin{abstract}
We present a scenario where dark matter is in the form of dark atoms that can accommodate the experimentally observed excess of positrons in PAMELA and AMS-02 while being compatible with the constraints imposed on the gamma-ray ux from Fermi/LAT. This scenario assumes that the dominant component of dark matter is in the form of a bound state between a helium nucleus and a -2 particle and a small component is in the form of a WIMP-like dark atom compatible with direct searches in underground detectors. One of the constituents of this WIMP-like state is a +2 metastable particle with a mass of $1 \mathrm{TeV}$ or slightly below that by decaying to $e^{+} e^{+}, \mu^{+} \mu^{+}$and $\tau^{+} \tau^{+}$produces the observed positron excess. These decays can naturally take place via GUT interactions. If it exists, such a metastable particle can be found in the next run of LHC. The model predicts also the ratio of leptons over baryons in the universe to be close to -3 .
\end{abstract}

\section{Introduction}

The possibility of dark matter being in the form of "dark atoms" has been studied extensively [1-21]. In this scenario, new stable particles are bound by new dark forces (like mirror partners of ordinary particles bound by mirror electromagnetism [22-26]). However, it turns out that even stable electrically charged particles can exist hidden in dark atoms, bound by ordinary Coulomb interactions (see [27-30] and references therein). Stable particles with charge -1 (and corresponding antiparticles as tera-particles [31]) are excluded due to overproduction of anomalous isotopes. However, negatively doubly charged particles are not constrained by anomalous isotope searches as much as -1 charged particles [32]. There exist several types of particle models where heavy stable -2 charged species, $\mathrm{O}^{--}$, are predicted:

(a) AC-leptons, predicted as an extension of the Standard Model, based on the approach of almost-commutative geometry [33-36];

(b) technileptons and antitechnibaryons in the framework of Walking Technicolor (WTC) [37-43].
All these models also predict corresponding +2 charge particles. If these positively charged particles remain free in the early universe, they can recombine with ordinary electrons in anomalous helium, which is strongly constrained in terrestrial matter. Therefore a cosmological scenario should provide a mechanism which suppresses anomalous helium. There are two possible mechanisms that can provide a suppression.

(i) The abundance of anomalous helium in the galaxy may be significant, but in terrestrial matter a recombination mechanism could suppress this abundance below experimental upper limits $[33,35]$. The existence of a new $U(1)$ gauge symmetry, causing new Coulomb-like long range interactions between charged dark matter particles, is crucial for this mechanism. This leads inevitably to the existence of dark radiation in the form of hidden photons.

(ii) Free positively charged particles are already suppressed in the early universe and the abundance of anomalous helium in the galaxy is negligible $[29,44]$. 
These two possibilities correspond to two different cosmological scenarios of dark atoms. The first one is realized in the scenario with AC leptons, forming neutral AC atoms [35]. The second assumes a charge asymmetry of the $\mathrm{O}^{--}$which forms the atom-like states with primordial helium $[29,44]$.

If new stable species belong to nontrivial representations of the $\mathrm{SU}(2)$ electroweak group, sphaleron transitions at high temperatures can provide the relation between baryon asymmetry and excess of -2 charge stable species, as it was demonstrated in the case of WTC [37, 45-47].

After formation in the Big Bang Nucleosynthesis (BBN), ${ }^{4} \mathrm{He}$ screens the $\mathrm{O}^{--}$charged particles in composite $\left({ }^{4} \mathrm{He}^{++} \mathrm{O}^{--}\right) \mathrm{OHe}$ "atoms" [44]. In all the models of $\mathrm{OHe}, \mathrm{O}^{--}$ behaves either as a lepton or as a specific "heavy quark cluster" with strongly suppressed hadronic interactions. Therefore $\mathrm{OHe}$ interactions with matter are determined by the nuclear interactions of He. These neutral primordial nuclear interacting objects can explain the modern dark matter density and represent a nontrivial form of strongly interacting dark matter [48-56].

The cosmological scenario of the $\mathrm{OHe}$ universe can explain many results of experimental searches for dark matter [29]. Such a scenario is insensitive to the properties of $\mathrm{O}^{--}$, since the main features of the $\mathrm{OHe}$ dark atoms are determined by their nuclear interacting helium shell. In terrestrial matter such dark matter species are slowed down and cannot cause significant nuclear recoil in the underground detectors, making them elusive in direct WIMP search experiments (where detection is based on nuclear recoil) such as CDMS, XENON100, and LUX [57-61]. The positive results of DAMA and possibly CRESST and CoGeNT experiments [62-66] can find in this scenario a nontrivial explanation due to a low energy radiative capture of $\mathrm{OHe}$ by intermediate mass nuclei $[29,30]$.

It has been also shown [37, 45-47] that a two-component dark atom scenario is also possible. Along with the dominant $\mathrm{O}^{--}$abundance, a much smaller excess of positively doubly charged techniparticles can be created. These positively charged particles are hidden in WIMP-like atoms, being bound to $\mathrm{O}^{--}$. In the framework of WTC such positively charged techniparticles can be metastable, with a dominant decay channel to a pair of positively charged leptons. In this paper we show that even a $10^{-6}$ fraction of such positively charged techniparticles with a mass of $1 \mathrm{TeV}$ or less and a lifetime of $10^{20} \mathrm{~s}$, decaying to $e^{+} e^{+}, \mu^{+} \mu^{+}$, and $\tau^{+} \tau^{+}$, can explain the observed excess of cosmic ray positrons, being compatible with the observed gamma-ray background.

One should note that, as it was shown in $[35,37,44$, 45] (for a review, see [29, 33] and references therein), the case of -2 charged stable particles is significantly different from the case of stable or metastable particles with charge -1 , avoiding severe constraints on charged particles from anomalous isotope searches and BBN due to their catalytic effects (see, e.g., [67-69]). In essence this difference comes from the fact that primordial He formed in BBN captures -2 charged particles in neutral $\mathrm{OHe}$ states, while -1 charged particles are captured by $\mathrm{He}$ in +1 charged ions, which either (if stable) form anomalous isotopes of hydrogen or (if longlived, but metastable) catalyze processes of light element production and influence their abundance. Nuclear physics of $\mathrm{OHe}$ is in the course of development, but a qualitative analysis has shown [46] that the OHe interactions with matter should not lead to overproduction of anomalous isotopes, while $\mathrm{OHe}$ catalytic effects in BBN can lead to primordial heavy element production, but not to overproduction of light elements.

The paper is organized as follows. In Section 2 we give a brief review of dark atoms made of stable charged techniparticles. In Section 3 we present the constraints and the predictions of the scenario with respect to the parameters of the Technicolor model we use, as well as how the ratio of lepton over baryon number is deduced. In Section 4 we show what GUT operators can implement the decay of the doubly charged particle to leptons. In Section 5, we show how the scenario of decaying dark matter can be realized, and how it can explain the PAMELA and AMS-02 results while satisfying the Fermi/LAT constraints. We conclude in Section 6.

\section{Dark Atoms from Techniparticles}

Technicolor theories that do not violate the electroweak precision tests, while not introducing large flavor changing currents, have been extensively studied lately (see [70] and references therein). Old models where fermions transformed under the fundamental representation of the gauge group required a large number of flavors (for a given number of colors) in order to be close to the conformal window and thus to suppress the flavor changing neutral currents. The need for many flavors coupled to the electroweak sector (that violates the electroweak precision measurements) disfavored Technicolor in the past. However, it has been demonstrated that once one allows fermions to transform under higher representations of the gauge group, quasi-conformality can be achieved even with a small number of colors and flavors [38-40]. This means that there is a set of Technicolor models that evade the strict constraints of the electroweak tests, making Technicolor a viable candidate for the $\mathrm{TeV}$ energy scale. Apart from the perturbative calculation of the oblique parameters [41] in this type of models, nonperturbative calculations based on holographic descriptions [71-73] showed that indeed the oblique $S$ parameter can be small. Note that the oblique parameters (e.g., $S, T$, and $U$ ) measure the modifications of the Standard Model gauge boson vacuum polarization amplitudes caused by contributions of new physics. These parameters are severely constrained by electroweak precision tests. Extra flavors that couple with the electroweak sector contribute to these parameters and can potentially exclude a model.

One of the simplest models that possesses the features described above, is the so-called Minimal Walking Technicolor $[38,42,74]$. The theory consists of two techniquarks transforming under the adjoint representation of an SU(2) gauge group, and an extra family of leptons $\nu^{\prime}$ and $\zeta$ coupled to the electroweak in order to cancel the global Witten anomaly. The hypercharge assignment can be chosen consistently (without introducing gauge anomalies) such that one of the techniquarks has zero electric charge. Such a simple theory can have a variety of dark matter candidates, ranging from 
dark matter particles that are Goldstone bosons of the theory (with nonzero technibaryon number) $[43,75,76]$ or Majorana WIMPs [77-82]. Apart from these possibilities, there is another intriguing scenario that is of an electromagnetic bound state between a +2 charged helium nucleus and a -2 charged techniparticle [37, 45]. More specifically in [37], we examined the possibility where the dark matter bound state is $\mathrm{He} \bar{U} \bar{U}$ or He $\zeta$. Recall that $U$ and $D$ are the two techniquarks of the theory and $\nu^{\prime}$ and $\zeta$ are the extra leptons. There is a gauge anomalous free hypercharge assignment where the charges of $U, D, v^{\prime}$, and $\zeta$ are, respectively, $+1,0,-1$, and -2 . We should also emphasize that, due to the fact that techniquarks transform under the adjoint representation of the gauge group, some of the Goldstone bosons are colorless diquarks (carrying technibaryon number). Apparently $\overline{U U}$ and $\zeta$ have charges -2 . This candidate $\operatorname{He} A$ (with $A$ being $\bar{U} \bar{U}$ or $\zeta$ ) is a Strongly Interacting Massive Particle (SIMP) rather than a WIMP due to the large geometric cross section of the helium component. Despite the large cross section, this candidate has not been ruled out by any experiment so far. Amazingly enough, although such a candidate interacts strongly with matter, it cannot be detected in earth based detectors (based on measuring the recoil energy) like CDMS, Xenon, or LUX. By the time such a particle reaches the detector, and it has lost most of its kinetic energy making it impossible to produce recoil energies above the detection threshold. In [45], we examined a generalized version of the aforementioned scenario, where although the majority of dark matter is $\mathrm{He} \bar{U} \bar{U}$ (or $\mathrm{He} \zeta$ ), a small component can be of the WIMP form $\bar{\zeta} \bar{U} \bar{U}$ (or $U U \zeta$ ). Such a WIMP component must be small since it is constrained by direct detection experiments.

In $[37,45]$, we had assumed that techniparticles are stable. In particular with respect to the technibaryons, the symmetry associated with the technibaryon number protected the lightest diquark Goldstone boson from decaying. Here we reexamine the scenario of [45] allowing decays of the techniparticles. It has been demonstrated that decaying dark matter can provide a possible explanation of the unexpected positron excess seen in PAMELA $[83,84]$. Decaying of dark matter particles through a dimension- 6 operator gives a lifetime

$$
\tau \sim 8 \pi \frac{M_{\mathrm{GUT}}^{4}}{m^{5}}=5 \times 10^{20} \mathrm{~s}\left(\frac{2 \mathrm{TeV}}{m}\right)^{5}\left(\frac{M_{\mathrm{GUT}}}{10^{15} \mathrm{GeV}}\right)^{4},
$$

where $m$ is the mass of the dark matter particle. Note that we have normalized the lifetime with respect to a GUT scale by an order of magnitude lower than the typical value of $2 \times 10^{16} \mathrm{GeV}$ suggested by supersymmetry. As we are going to argue a small component of dark matter with a mass of $\sim \mathrm{TeV}$ or less and a lifetime of $10^{20} \mathrm{~s}$ can accommodate nicely the positron excess seen in PAMELA and AMS-02 data. In addition such a lifetime is sufficiently large in order not to deplete the density of this component of dark matter by today since it is a few orders of magnitude larger than the age of the universe. As it was stressed in [83], dimension-6 operators are very natural objects in Technicolor, and therefore such a framework becomes very appealing.

\section{Techniparticle Excess}

We already mentioned that the MWT has two techniquarks $U$ and $D$ in the adjoint representation of the Technicolor SU(2) with charges +1 and 0 and two new leptons $\nu^{\prime}$ and $\zeta$ with charges -1 and -2 , respectively. The theory possesses a global $\mathrm{SU}(4)$ symmetry that breaks spontaneously to an $\mathrm{SO}(4)$. Out of the 9 Goldstone bosons, three of them (with the quantum numbers of the usual pions) are eaten by the $W$ and $Z$ bosons, while the rest 6 are the colorless diquarks $U U, U D$, and $D D$ and their antiparticles [43].

We are going to consider two possibilities. The first one is to have an excess of -2 charge $\bar{U} \bar{U}$ and a little of $+2 \bar{\zeta}$. The main component of dark matter is the SIMP He $\bar{U} \bar{U}$. There is also a small WIMP component of $\bar{\zeta} \bar{U} \bar{U}$. The second scenario is to have an excess of $\zeta$ and a little of $U U$, in such a way that the main SIMP component of dark matter is $\mathrm{He} \zeta$ and the small WIMP one is $U U \zeta$. In both cases we have assumed that $U U$ is the lightest among the technibaryons and similarly $\zeta$ is the lightest of the new leptons. The calculation of the relic density of the technibaryons taking into account sphaleron violating processes, weak equilibration, and overall charge neutrality gives similarly to [43]

$$
\frac{\mathrm{TB}}{B}=-\sigma_{U U}\left(\frac{L^{\prime}}{B} \frac{1}{3 \sigma_{\zeta}}+1+\frac{L}{3 B}\right),
$$

where TB, $B, L$, and $L^{\prime}$ are the technibaryon, baryon, lepton, and new lepton family number, respectively. $\sigma_{i}$ are statistical factors for the specific particle $i$ given by

$$
\sigma_{i}= \begin{cases}6 f\left(\frac{m_{i}}{T^{*}}\right) & \text { for fermions, } \\ 6 g\left(\frac{m_{i}}{T^{*}}\right) & \text { for bosons, }\end{cases}
$$

where the functions $f$ and $g$ are defined as follows:

$$
\begin{aligned}
& f(z)=\frac{1}{4 \pi^{2}} \int_{0}^{\infty} d x x^{2} \cosh ^{-2}\left(\frac{1}{2} \sqrt{x^{2}+z^{2}}\right), \\
& g(z)=\frac{1}{4 \pi^{2}} \int_{0}^{\infty} d x x^{2} \sinh ^{-2}\left(\frac{1}{2} \sqrt{x^{2}+z^{2}}\right) .
\end{aligned}
$$

$T^{*}$ is the freeze-out temperature for the sphaleron process, usually taken somewhere between 150 and $250 \mathrm{GeV}$. In the first aforementioned possibility, the dark matter density is

$$
\begin{aligned}
\frac{\Omega_{d}}{\Omega_{B}} & =\frac{\Omega_{\bar{\zeta} \bar{U} \bar{U}}}{\Omega_{B}}+\frac{\Omega_{\mathrm{He} \bar{U} \bar{U}}}{\Omega_{B}} \\
& =\left|\frac{L^{\prime}}{B}\right| \frac{m_{d}}{m_{p}}+\left(\frac{3}{2}\left|\frac{\mathrm{TB}}{B}\right|-\left|\frac{L^{\prime}}{B}\right|\right) \frac{m_{s}}{m_{p}}=5.47,
\end{aligned}
$$

where $m_{d}, m_{s}$, and $m_{p}$ are the masses of $U U \zeta, \mathrm{He} \bar{U} \bar{U}$, and proton, respectively. We have taken the ratio of dark matter to baryonic matter to be $\sim 5.47$. If $\xi$ denotes the fraction of the WIMP component $(\bar{\zeta} \bar{U} \bar{U})$ of dark matter, then the ratio of leptons over baryons is given by

$$
\frac{L}{B}=-3+5.47 m_{p}\left[\frac{\xi}{m_{d} \sigma_{\zeta}}+\frac{2 \xi}{m_{d} \sigma_{U U}}+\frac{2(1-\xi)}{m_{s} \sigma_{U U}}\right] .
$$




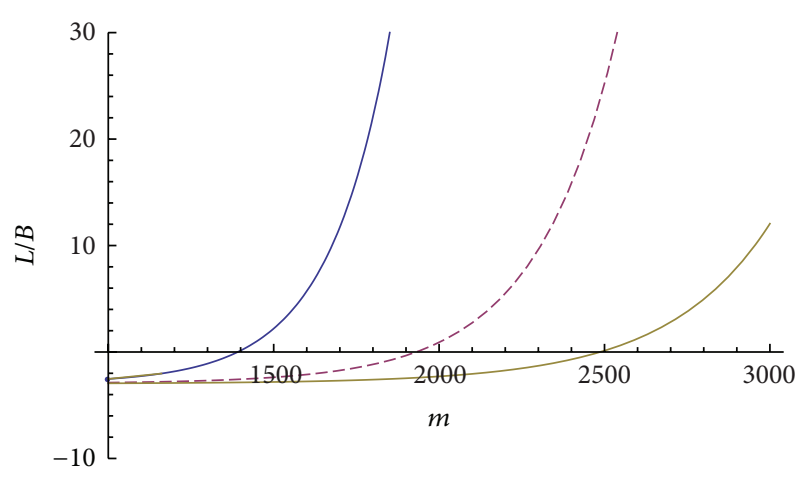

(a)

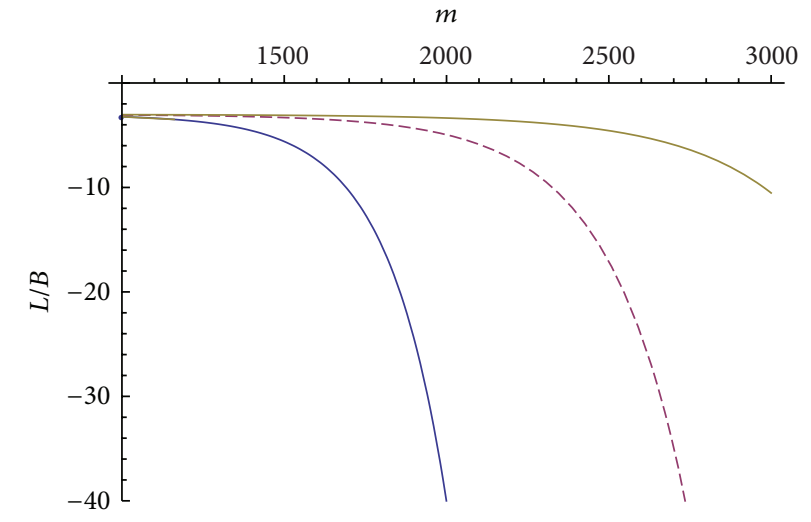

(b)

FIGURE 1: The ratio $L / B$ for the two scenarios explained in the text: (6) and (8), respectively, for $m=m_{U U}=m_{\zeta}$ (in GeV) and $\xi=10^{-6}$. The three different lines, that is, thin solid, dashed, and thick solid, correspond to freeze-out temperature for the sphalerons $T^{*}$ of 150,200 , and $250 \mathrm{GeV}$, respectively.

In the second scenario (that of $\mathrm{He} \zeta$ and $U U \zeta$ ),

$$
\frac{\Omega_{d}}{\Omega_{B}}=\frac{\Omega_{U U \zeta}}{\Omega_{B}}+\frac{\Omega_{\mathrm{He} \zeta}}{\Omega_{B}}=\frac{3}{2} \frac{T B}{B} \frac{m_{d}}{m_{p}}+\left(\frac{L^{\prime}}{B}-\frac{3}{2} \frac{T B}{B}\right) \frac{m_{s^{\prime}}}{m_{p}},
$$

where $m_{s}^{\prime}$ is the mass of $\mathrm{He} \zeta$. The ratio $L / B$ is

$$
\frac{L}{B}=-3-5.47 m_{p}\left(\frac{\xi}{m_{d} \sigma_{\zeta}}+\frac{2 \xi}{m_{d} \sigma_{U U}}+\frac{1-\xi}{m_{s^{\prime}} \sigma_{\zeta}}\right) .
$$

$\xi$ here is again the fraction of the WIMP-like component of dark matter. There are two points we would like to emphasize. The first one is that both possibilities give a ratio of lepton over baryon numbers very close to -3 if the masses of $U U$ and $\zeta$ remain around $1 \mathrm{TeV}$. In fact the first scenario gives a ratio slightly above -3 and the second gives a ratio slightly below. $L / B$ starts deviating (exponentially) as a function of the mass of $U U$ and/or $\zeta$ once we go to masses much higher than 1.5 to $2 \mathrm{TeV}$ (see Figure 1). The second point we would like to stress is that $\xi$ is constrained by earth based direct detection search experiments. In [45] we found that the WIMP component of this dark matter scenario cannot be more than $1 \%$ (or $\xi<0.01)$. Since then, the constraint from the CDMS and Xenon experiments has improved significantly and more severe constraints from LUX appeared. The cross section of $U U \zeta$ (or its antiparticle) with a proton is [85]

$$
\sigma_{p}=\frac{G_{F}^{2}}{2 \pi} \mu^{2} \bar{Y}^{2} F^{2} \simeq 1.8 \times 10^{-39} \mathrm{~cm}^{2},
$$

where $\bar{Y}=Y_{L}+Y_{R}$, that is, the sum of the hypercharge of left and right components. It is easy to check that in our case $\bar{Y}=-1 / 2$. This is because $U U$ has $Y_{L}=1$ and $Y_{R}=2$ and $\zeta$ has $Y_{L}=-3 / 2$ and $Y_{R}=-2$. The total sum is $-1 / 2$. In addition since both $U U$ and $\zeta$ are much heavier than the proton, the reduced mass $\mu$ is approximately the mass of the proton. The form factor $F$ depends on the target nucleus and the recoil energy. For example, for Ge detector with recoil energies between 20 and $50 \mathrm{keV}$, the form factor ranges from 0.43 to 0.72 [43]. Here in this estimate of the WIMP-proton cross section we have set $F=1$. The results of the LUX experiment [61] exclude WIMPs with a cross section $10^{-45} \mathrm{~cm}^{2}$ for a typical WIMP mass of $1 \mathrm{TeV}$. This means that WIMPs with the cross section of $U U \zeta$ can make up only a component of $\sim 10^{-6}$ or smaller of the total dark matter. Here we are going to use a typical value of $\xi=10^{-6}$.

\section{Decaying Dark Matter}

As we mentioned in the previous section, we might have a $\sim 10^{-6}$ (or less) WIMP component in our dark matter framework. This comes in the form of $\bar{\zeta} \bar{U} \bar{U}$ (first scenario) or $U U \zeta$ (second scenario). Our goal is to consider decay processes that can produce the excess of positrons seen in PAMELA and AMS-02. For this, it is generically better if the +2 objects decay accordingly.

In the first considered scenario we assume that $\bar{U} \bar{U}$ is stable, and therefore the SIMP component which consists the overwhelming part of dark matter is unaffected. On the other hand, we assume that $\bar{\zeta}^{++}$can decay to leptons. By construction since $\zeta$ and $\nu^{\prime}$ belong to the same electroweak doublet, $\zeta$ couples to $\nu^{\prime}$ and $W^{-}$. Since $\nu^{\prime}$ is a lepton with an electric charge -1 , it can in principle slightly mix with the usual -1 leptons, that is, electrons, muons, and taus. The tiny WIMP component of dark matter made of $\bar{\zeta} \bar{U} \bar{U}$ decays due to the fact that $\bar{\zeta}$ can decay to a $W^{+}$and (via $\left.\bar{\nu}^{\prime}\right)$ to positrons, antimuons, and antitaus. We assume that $\nu^{\prime}$ is heavier than $\zeta$, and therefore the decay is suppressed. In order not to get very fast decays of $\bar{\zeta}$, the mixing of $\bar{\nu}^{\prime}$ with positrons and so forth has to be extremely small. However, this is something expected due to experimental constraints as well as due to the fact that $v^{\prime}$ is much heavier than the leptons. It is also expected that the mixing between $\nu^{\prime}$ and $\tau$ would be larger than $\nu^{\prime}$ and $\mu$ or $\nu^{\prime}$ and positrons. The decay in this scenario can be accommodated via a dimension -5 operator. However, 
decays of $\bar{\zeta}$ to positrons, $\mu^{+} \mu^{+}$, or $\tau^{+} \tau^{+}$can lead to unwanted production of hadrons via decays of $W^{+}$. Therefore we focus on the second case.

In the second scenario the small WIMP component is made of $U U \zeta$. In this case we assume that $\zeta$ is stable (and no mixing with other leptons exists), but the $U U$ Goldstone boson decays via a GUT interaction. A natural dimension-6 operator that can accommodate the decay can be of the form

$$
\mathcal{O}=\frac{U^{T} C U \psi^{T} C^{\prime} \psi}{\Lambda_{\mathrm{GUT}}^{2}}
$$

where $\psi$ is an electron, muon, or tau. Notice that, due to the transpose instead of the bar, such an operator violates both the lepton and the technibaryon number. It allows a possible decay of $U U$ to two positrons (or two antimuons or antitaus (in principle we can have an even more general operator where $U U$ decays to different species of antileptons, i.e., a positron, an antimuon, etc)):

$$
U U \longrightarrow e^{+}+e^{+} \text {. }
$$

It is understood that although $C$ and $C^{\prime}$ can be generic Dirac matrices, $C$ has to be the charge conjugate matrix in order for $U^{T} C U$ to be the pseudo-Goldstone boson $U U$. If we require that parity is not violated by the interaction, $C^{\prime}$ must also be the charge conjugate matrix. In case parity is violated, $C^{\prime}$ can be $C \gamma_{5}$ (as it is a well-known fact that $\psi^{T} C \gamma_{5} \psi$ is a scalar). Of course nothing forbids a similar decay of $U U$ to two quarks or even a quark and a lepton, as it would depend on the details of the GUT interaction. However, here we do not want to speculate regarding the GUT interactions but simply to demonstrate that such a realization can in fact produce the positron spectrum seen by experiments. As we already mentioned, a dimension- 6 operator of the above form would give according to (1) a lifetime of the order of $10^{20} \mathrm{~s}$ for a mass of $U U$ of the order of TeV. If $U U$ does not decay to hadrons, this scenario is more appropriate for explaining the positron excess compared to the first scenario we mentioned because in the first scenario the decay of $\bar{\zeta}$ will always be accompanied by hadronic decays that are not seen by PAMELA.

\section{Positron Excess and Fit to the PAMELA and AMS-02 Data}

Here we show the impact of decaying $U U$ particles on the cosmic positron flux and diffuse gamma radiation. The socalled "PAMELA anomaly" in the cosmic positron spectrum [86] has been recently confirmed also by AMS-02 [87]. This anomaly cannot be explained by positrons of only secondary origin, and therefore primary positron sources are needed to explain the data. There are attempts to realize it based on decaying or annihilating dark matter models. Any scenario that provides positron excess is constrained by other observational data mainly from the data on cosmic antiprotons, gamma-radiation from our halo (diffuse gamma-background), and other galaxies and clusters [8895]. If dark matter does not produce antiprotons, then

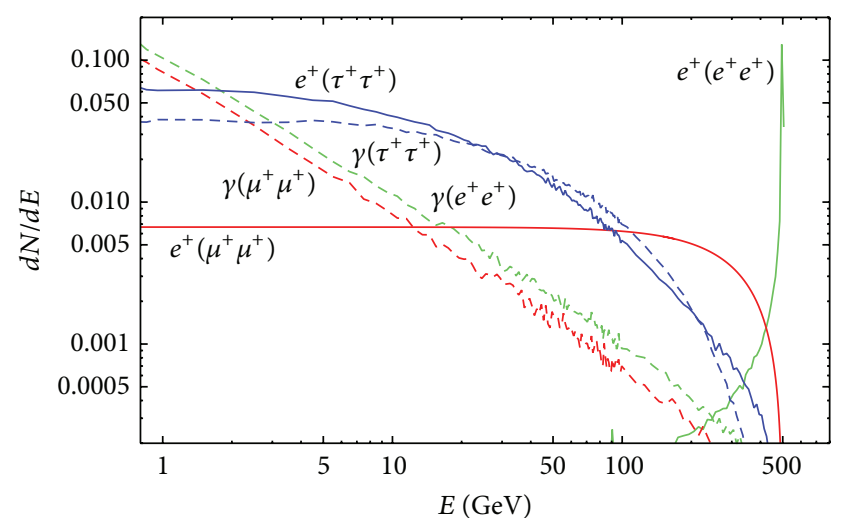

FIGURE 2: Spectra of gamma-rays and positrons from decays $U U \rightarrow$ $e^{+} e^{+}, \mu^{+} \mu^{+}, \tau^{+} \tau^{+}$. We used Pythia 6.4 [97].

the diffuse gamma-ray background gives the most stringent and model-independent constraints.

In our scenario the $U U$ component of a tiny UU $\zeta$ WIMP component of dark matter decays as $U U \rightarrow e^{+} e^{+}, \mu^{+} \mu^{+}, \tau^{+} \tau^{+}$ in principle with different branching ratios. All decay modes give directly or through intermediate particle decays the positrons and photons, which are hereafter referred as final state radiation (FSR). In Figure 2 we show the produced positron and gamma spectra for each decay mode individually. Note that, unlike PAMELA, the AMS-02 disfavors decays purely to $e^{+}$and $\mu^{+}$(although it does not exclude them).

In the context of indirect dark matter searches from cosmic rays (CR), the leptonic decay modes have been studied extensively (see, e.g., [88-95]), using a variety of different approaches in estimating the CR signals. For our estimate, we adopt the following model of positron propagation in the galaxy. Due to energy losses, positrons have a finite diffusion length at given energy $E$

$$
\lambda \sim \sqrt{\int D d t}=\sqrt{\int D \frac{d E}{b}} \sim 10 \mathrm{kpc} \sqrt{E^{-0.7}-E_{0}^{-0.7}},
$$

where $D \approx 4 \cdot 10^{28} \mathrm{~cm}^{2} \mathrm{~s}^{-1} E^{0.3}$ is a typical value for the diffusion coefficient [96], $b=\beta E^{2}$ is the rate of energy losses with $\beta \sim 10^{-16} \mathrm{~s}^{-1} \mathrm{GeV}^{-1}$, and $E_{0}$ is the initial energy. All energies are measured in $\mathrm{GeV}$. The effect of the diffusion in the propagation can be estimated by assuming a homogeneous distribution of the sources. In fact, the result of diffusion is not sensitive to the effects of inhomogeities, because it depends on the averaged density within the diffusion length. Since we are interested in positron energies above $\sim 10 \mathrm{GeV}$, which corresponds to $\lambda \lesssim 5 \mathrm{kpc}$ (see (12)) over which no essential inhomogeneity effects are expected, this simple approximation we make here is good. At $E \lesssim$ $10 \mathrm{GeV}$, secondary positrons dominate the spectrum. If $\lambda$ exceeds the size of the magnetic halo $(\mathrm{MH})(h \sim 4 \mathrm{kpc}$ in height and $R \sim 15 \mathrm{kpc}$ in width), the leakage of particles from the halo should be taken into account. We consider this effect 
by introducing a suppression factor, which is equal to the ratio of the volume of $\mathrm{MH}$ contained within the sphere of radius $\lambda$ :

$Q=1-\frac{(\lambda-h)^{2}(2 \lambda+4)}{2 \lambda^{3}} \eta(\lambda-h)-\frac{2 h\left(\lambda^{2}-r^{2}\right)}{3 \lambda^{3}} \eta(\lambda-R)$,

where $\eta$ is the step function. If $d N / d E_{0}$ is the number of positrons produced in a single decay (see Figure 2), the positron flux near the Earth can be estimated as

$$
F(E)=\frac{c}{4 \pi} \frac{n_{\mathrm{loc}}}{\tau} \frac{1}{\beta E^{2}} \int_{E}^{m / 2} \frac{d N}{d E_{0}} Q\left(\lambda\left(E_{0}, E\right)\right) d E_{0},
$$

where $n_{\text {loc }}=\xi \cdot\left(0.3 \mathrm{GeV} / \mathrm{cm}^{3}\right) m_{U U}^{-1}$ is the local number density of $U U$ particles with $\xi=10^{-6} \xi_{-6}$. Recall that $\xi$ is the fraction of dark matter in the WIMP UU $\zeta$ component.

The effect of solar modulation becomes important at the less interesting low energy part of the positron spectrum. To account for this effect, we have adopted the forced field model [98] with two different $\phi$ parameters for positrons and electrons. They are easily adjusted so they can fit the data points at low energy. The positron and electron background components were taken from [99]. In Figure 3 we present the positron excess due to $U U$ decays for two values of the mass of $U U, m_{U U}=0.7 \mathrm{TeV}$ and $m_{U U}=1 \mathrm{TeV}$. We also show the lifetime of $U U \tau$ and the branching ratios that fit the experimental data optimally for each choice of $m_{U U}$. They evade the existing constraints of [88-95].

The gamma-ray flux from $U U$ decays has two main contributions: one from FSR (shown in Figure 2) and another one from Inverse Compton (IC) scattering of positrons on background photons (star light, infrared background, and $\mathrm{CMB})$.

For the FSR photons produced by $U U$ decays in our galaxy, the flux arriving in the Earth is given by

$$
F_{\mathrm{FSR}}=\frac{n_{\mathrm{loc}}}{\tau} \frac{1}{4 \pi \Delta \Omega_{\mathrm{obs}}} \int_{\Delta \Omega_{\mathrm{obs}}} \frac{n(r)}{n_{\mathrm{loc}}} d l d \Omega \cdot \frac{d N_{\gamma}}{d E},
$$

where we use an isothermal profile $n(r) / n_{\text {loc }}=\left((5 \mathrm{kpc})^{2}+\right.$ $\left.(8.5 \mathrm{kpc})^{2}\right) /\left((5 \mathrm{kpc})^{2}+r^{2}\right), r$ and $l$ are the distances from the Galactic center and the Earth, respectively. We obtain the averaged flux over the solid angle $\Delta \Omega_{\text {obs }}$ corresponding to $|b|>10^{\circ}, 0<l<360^{\circ}$. For the IC photons from our galaxy, we have estimated the contribution following [100]. In Figure 4 we show both contributions in the gamma-ray flux for the same parameters as in Figure 3.

Decays of $U U$, which are outside our Galaxy being homogeneously distributed over the Universe, should also contribute to the observed gamma-ray flux. For FSR photons this contribution can be estimated as

$$
\begin{aligned}
F_{\mathrm{FSR}}^{(U)}(E)= & \frac{c}{4 \pi} \frac{\left\langle n_{\bmod }\right\rangle}{\tau} \int \frac{d N}{d E} d t=\frac{c\left\langle n_{\bmod }\right\rangle}{4 \pi \tau} \\
& \times \int_{0}^{\min (1100,(m / 2 E)+1)} \frac{d N}{d E_{0}}\left(E_{0}=E(z+1)\right) \\
& \times \frac{H_{\bmod }^{-1} d z}{\sqrt{\Omega_{\Lambda}+\Omega_{m}(z+1)^{3}}},
\end{aligned}
$$

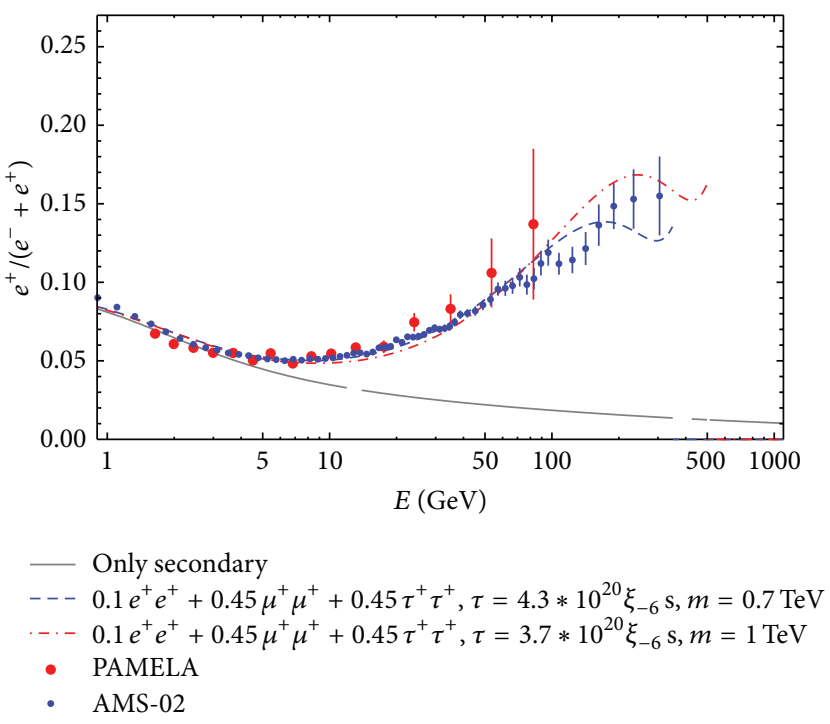

Figure 3: Positron excess due to $U U \rightarrow e^{+} e^{+}, \mu^{+} \mu^{+}, \tau^{+} \tau^{+}$decays compared to PAMELA and AMS-02 data.

where $z=1100$ corresponds to the recombination epoch, $\left\langle n_{\bmod }\right\rangle$ is the current cosmological number density of $U U$, $H_{\text {mod }}^{-1}=(3 / 2) t$ mod $\sqrt{\Omega_{\Lambda}} \ln \left(\left(1+\sqrt{\Omega_{\Lambda}}\right) / \sqrt{\Omega_{m}}\right)$ is the inverse value of the Hubble parameter with $t_{\bmod }$ being the age of the universe, and $\Omega_{\Lambda}$ and $\Omega_{m}=1-\Omega_{\Lambda}$ are, respectively, the current vacuum and matter relative densities. Note in (16) the transition between distributions at different $z, d N / d E \rightarrow$ $\left(d N / d E_{0}\right)(z+1)$. This extragalactic contribution to FSR increases significantly the total gamma-ray flux as shown in Figure 4 by dot-dashed lines.

It is not expected that extragalactic IC photons can contribute significantly to the spectrum. Indeed, mainly only low energetic CMB photons are present in the medium outside the galaxy (or before the galactic stage). After the scattering of electrons with energy $E_{0} \lesssim 500 \mathrm{GeV}$ off $\mathrm{CMB}$ photons with energy $\omega_{\mathrm{CMB}} \lesssim 10^{-3}(z+1) \mathrm{eV}$, the recoiled photons acquire at redshift $z$ energy $\omega \sim\left(E_{0} / m_{e}\right)^{2} \omega_{\mathrm{CMB}} \lesssim$ $(z+1) \mathrm{GeV}$, which is below $1 \mathrm{GeV}$ in the modern epoch. It makes therefore this contribution indifferent for the energy range of Fermi/LAT.

To conclude, on the basis of Figure 4, one may assert that the considered scenarios of $U U$ decays satisfy the Fermi/LAT constraints. In addition, although we used the best fit values for the branching ratios, we have found that some small variation of the branching ratios is possible. If one chooses $m_{U U}>1 \mathrm{TeV}$, a possible satisfaction of the constraints is possible at the expense of the positron spectrum fit.

\section{Conclusions}

Dark matter can potentially be in the form of neutral $\mathrm{OHe}$ dark atoms made of stable heavy doubly charged particles and primordial He nuclei bound by ordinary Coulomb interactions. This scenario sheds new light on the nature of dark matter and offers a nontrivial solution for the puzzles 

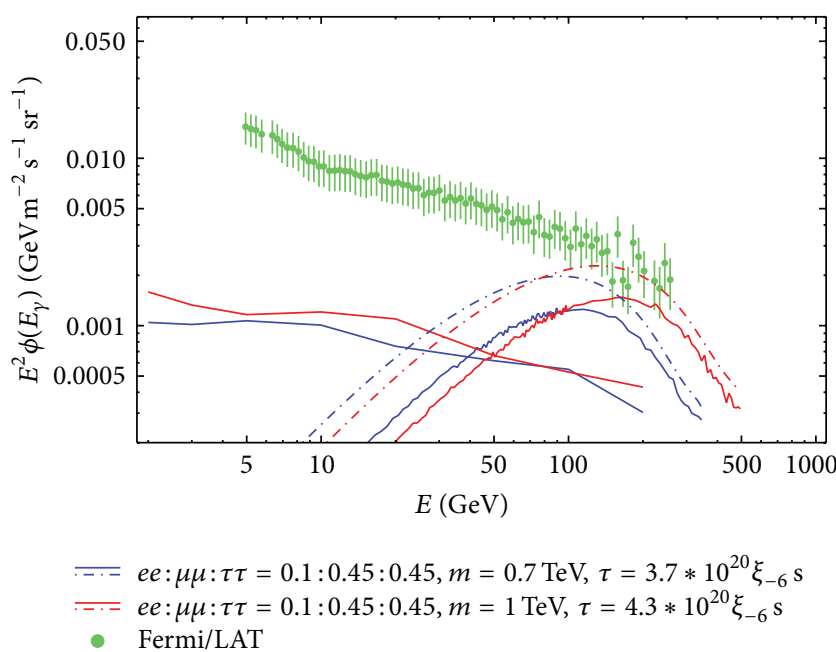

FIgURE 4: Gamma-ray flux from UU decays in the galaxy $(|b| \geq$ $10^{\circ}$ ) in comparison to the Fermi/LAT data on diffuse background [101]. Two contributions are shown: IC (left curves) and FSR (right curves). Dot-dashed curves take into account FSR photons of both galactic and extragalactic origin.

of direct dark matter searches. It can be realized in the framework of Minimal Walking Technicolor, in which an exact relation between the dark matter density and baryon asymmetry can be naturally obtained predicting also the ratio of leptons over baryons in the universe. In the context of this scenario a sparse component of WIMP-like dark atoms of charged techniparticles can also appear. Direct searches for WIMPs put severe constraints on the presence of this component. However, we demonstrated in this paper that the existence of a metastable positively doubly charged techniparticle, forming this tiny subdominant WIMP-like dark atom component and satisfying the direct WIMP searches constraints, can play an important role in the indirect effects of dark matter. We found that decays of such positively charged constituents of WIMP-like dark atoms to the leptons $e^{+} e^{+}, \mu^{+} \mu^{+}$, and $\tau^{+} \tau^{+}$can explain the observed excess of high energy cosmic ray positrons, while being compatible with the observed gamma-ray background. These decays are naturally facilitated by GUT scale interactions. This scenario makes a prediction about the ratio of leptons over baryons in the universe to be close to -3 . The best fit of the data takes place for a mass of this doubly charged particle of $1 \mathrm{TeV}$ or below making it accessible in the next run of LHC.

\section{Conflict of Interests}

The authors declare that there is no conflict of interests regarding the publication of this paper.

\section{Acknowledgment}

C. Kouvaris is supported by the Danish National Research Foundation, Grant no. DNRF90.

\section{References}

[1] S. I. Blinnikov and M. Yu. Khlopov, "On possible effects of mirror particles," Soviet Journal of Nuclear Physics, vol. 36, p. 472, 1982.

[2] S. I. Blinnikov and M. Y. Khlopov, "Excitation of the solar oscillations by objects consisting of y-matter," Solar Physics, vol. 82, no. 1-2, pp. 383-385, 1983.

[3] M. Yu. Khlopov, G. M. Beskin, N. E. Bochkarev, L. A. Pustylnik, and S. A. Pustylnik, "Observational physics of mirror world," Soviet Astronomy, vol. 35, no. 21, 1991.

[4] H. M. Hodges, "Mirror baryons as the dark matter," Physical Review D, vol. 47, no. 2, pp. 456-459, 1993.

[5] H. Goldberg and L. J. Hall, "A new candidate for dark matter," Physics Letters B, vol. 174, no. 2, pp. 151-155, 1986.

[6] Z. G. Berezhiani and R. N. Mohapatra, "Reconciling present neutrino puzzles: sterile neutrinos as mirror neutrinos," Physical Review D, vol. 52, no. 11, pp. 6607-6611, 1995.

[7] Z. G. Berezhiani, A. D. Dolgov, and R. N. Mohapatra, "Asymmetric inflationary reheating and the nature of mirror universe," Physics Letters B, vol. 375, no. 1-4, pp. 26-36, 1996.

[8] R. Foot and R. R. Volkas, "Neutrino physics and the mirror world: how exact parity symmetry explains the solar neutrino deficit, the atmospheric neutrino anomaly, and the LSND experiment," Physical Review D, vol. 52, no. 11, pp. 6595-6606, 1995.

[9] R. N. Mohapatra and V. L. Teplitz, "Mirror dark matter and galaxy core densities," Physical Review D, vol. 62, no. 6, Article ID 063506, 5 pages, 2000.

[10] R. Foot, "Mirror matter-type dark matter," International Journal of Modern Physics D, vol. 13, no. 10, pp. 2161-2192, 2004.

[11] M. J. Strassler and K. M. Zurek, "Echoes of a hidden valley at hadron colliders," Physics Letters B, vol. 651, no. 5-6, pp. 374379, 2007.

[12] N. Arkani-Hamed, D. P. Finkbeiner, T. R. Slatyer, and N. Weiner, "A theory of dark matter," Physical Review D, vol. 79, no. 1, Article ID 015014, 2009.

[13] D. E. Kaplan, G. Z. Krnjaic, K. R. Rehermann, and C. M. Wells, "Atomic dark matter," Journal of Cosmology and Astroparticle Physics, vol. 2010, p. 021, 2010.

[14] J. G. Wacker, S. R. Behbahani, M. Jankowiak, and T. Rube, "Nearly supersymmetric dark atoms," Advances in High Energy Physics, vol. 2011, Article ID 709492, 2011.

[15] D. E. Kaplan, G. Z. Krnjaic, K. R. Rehermannc, and C. M. Wells, "Dark atoms: asymmetry and direct detection," Journal of Cosmology and Astroparticle Physics, vol. 2011, p. 011, 2011.

[16] J. M. Cline, Z. Liu, and W. Xue, "Millicharged atomic dark matter," Physical Review D, vol. 85, Article ID 101302(R), 2012.

[17] J. M. Cline, Z. Liu, and W. Xue, "Optimistic CoGeNT analysis," Physical Review D, vol. 87, Article ID 015001, 2013.

[18] F.-Y. Cyr-Racine and K. Sigurdson, "Cosmology of atomic dark matter," Physical Review D, vol. 87, Article ID 103515, 2013.

[19] F.-Y. Cyr-Racine, R. de Putter, A. Raccanelli, and K. Sigurdson, "Constraints on large-scale dark acoustic oscillations from cosmology," Physical Review D, vol. 89, Article ID 063517, 2014.

[20] D. H. Weinberg, J. S. Bullock, F. Governato, R. Kuzio de Naray, and A. H. G. Peter, "Cold dark matter: controversies on small scales," submitted to Proceedings of the National Academy of Sciences of the United States of America, http://arxiv.org/ abs/1306.0913. 
[21] J. M. Cline, Z. Liu, G. D. Moore, and W. Xue, "Scattering properties of dark atoms and molecules," Physical Review D, vol. 89, Article ID 043514, 2014.

[22] T. D. Lee and C. N. Yang, "Question of parity conservation in weak interactions," Physical Review, vol. 104, no. 1, pp. 254-258, 1956.

[23] I. Yu. Kobzarev, L. B. Okun, and I. Ya. Pomeranchuk, Soviet Journal of Nuclear Physics, vol. 3, p. 837, 1966.

[24] B. Ya. Zel'dovich and M. Yu. Khlopov, "The neutrino mass in elementary-particle physics and in big bang cosmology," Soviet Physics Uspekhi, vol. 24, p. 755, 1981.

[25] L. B. Okun, "Mirror particles and mirror matter: 50 years of speculation and searching," Physics-Uspekhi, vol. 50, no. 4, pp. 380-389, 2007.

[26] P. Ciarcelluti, "Cosmology with mirror dark matter," International Journal of Modern Physics D, vol. 19, no. 14, pp. 2151-2230, 2010.

[27] M. Y. Khlopov, A. G. Mayorov, and E. Y. Soldatov, "Puzzles of dark matter in the light of dark atoms," Journal of Physics: Conference Series, vol. 309, no. 1, Article ID 012013, 2011.

[28] M. Y. Khlopov, A. G. Mayorov, and E. Y. Soldatov, Bled Workshops in Physics, vol. 11, p. 73, 2010.

[29] M. Y. Khlopov, "Physics of dark matter in the light of dark atoms," Modern Physics Letters A, vol. 26, no. 38, pp. 2823-2839, 2011.

[30] M. Yu. Khlopov, "Fundamental particle structure in the cosmological dark matter," International Journal of Modern Physics A, vol. 28, no. 29, Article ID 1330042, 2013.

[31] S. L. Glashow, "A sinister extension of the standard model to $S U(3) X S U(2) X S U(2) X U(1), " \quad$ http://arxiv.org/abs/hep$\mathrm{ph} / 0504287$.

[32] D. Fargion and M. Yu. Khlopov, “Tera-leptons' shadows over Sinister universe," Gravitation and Cosmology, vol. 19, pp. 219231, 2013.

[33] M. Y. Khlopov, "New symmetries in microphysics, new stable forms of matter around us," Annales de la Fondation Louis de Broglie, vol. 31, no. 2-3, pp. 257-272, 2006.

[34] C. A. Stephan, "Almost-commutative geometries beyond the standard model," Journal of Physics A, vol. 39, p. 9657, 2006.

[35] M. Y. Khlopov, C. A. Stephan, and D. Fargion, "Dark matter with invisible light from heavy double charged leptons of almostcommutative geometry?" Classical and Quantum Gravity, vol. 23, no. 24, pp. 7305-7354, 2006.

[36] A. Connes, Noncommutative Geometry, Academic Press, San Diego, Calif, USA, 1994.

[37] M. Y. Khlopov and C. Kouvaris, "Strong interactive massive particles from a strong coupled theory," Physical Review D, vol. 77, no. 6, Article ID 065002, 2008.

[38] F. Sannino and K. Tuominen, "Orientifold theory dynamics and symmetry breaking," Physical Review D, vol. 71, no. 5, pp. 1-4, 2005.

[39] D. K. Hong, S. D. H. Hsu, and F. Sannino, "Composite Higgs from higher representations," Physics Letters B, vol. 597, no. 1, pp. 89-93, 2004.

[40] D. D. Dietrich, F. Sannino, and K. Tuominen, "Light composite Higgs boson from higher representations versus electroweak precision measurements: predictions for CERN LHC," Physical Review D, vol. 72, no. 5, Article ID 055001, 2005.

[41] D. D. Dietrich, F. Sannino, and K. Tuominen, "Light composite Higgs and precision electroweak measurements on the $\mathrm{Z}$ resonance: an update," Physical Review D, vol. 73, no. 3, Article ID 037701, 2006.

[42] S. B. Gudnason, C. Kouvaris, and F. Sannino, "Towards working technicolor: effective theories and dark matter," Physical Review D, vol. 73, no. 11, Article ID 115003, 2006.

[43] S. B. Gudnason, C. Kouvaris, and F. Sannino, "Dark matter from new technicolor theories," Physical Review D, vol. 74, no. 9, Article ID 095008, 2006.

[44] M. Y. Khlopov, "Composite dark matter from the fourth generation," JETP Letters, vol. 83, no. 1, pp. 1-4, 2006.

[45] M. Y. Khlopov and C. Kouvaris, "Composite dark matter from a model with composite Higgs boson," Physical Review D, vol. 78, no. 6, Article ID 065040, 2008.

[46] M. Y. Khlopov, “The puzzles of dark matter searches," in Invisible Universe International Conference, vol. 1241 of AIP Conference Proceedings, pp. 388-397, July 2009.

[47] M. Y. Khlopov, A. G. Mayorov, and E. Y. Soldatov, "Composite dark matter and puzzles of dark matter searches," International Journal of Modern Physics D, vol. 19, no. 8-10, pp. 1385-1395, 2010.

[48] B. D. Wandelt, R. Dave, G. R. Farrar, P. C. McGuire, D. N. Spergel, and P. J. Steinhardt, "Self-Interacting dark matter," in Proceedings of the 4th International Symposium on Sources and Dete Conference, pp. 263-274, 2000.

[49] P. C. McGuire and P. J. Steinhardt, "Cracking open the window for strongly interacting massive particles as the halo dark matter," in Proceedings of the International Cosmic Ray Conference, p. 1566, Hamburg, Germany, 2001.

[50] G. Zaharijas and G. R. Farrar, "Window in the dark matter exclusion limits," Physical Review D, vol. 72, no. 8, Article ID 083502, 2005.

[51] C. B. Dover, T. K. Gaisser, and G. Steigman, "Cosmological constraints on new stable hadrons," Physical Review Letters, vol. 42, no. 17, pp. 1117-1120, 1979.

[52] S. Wolfram, "Abundances of new stable particles produced in the early universe," Physics Letters B, vol. 82, no. 1, pp. 65-68, 1979.

[53] G. D. Starkman, A. Gould, R. Esmailzadeh, and S. Dimopoulos, "Opening the window on strongly interacting dark matter," Physical Review D, vol. 41, no. 12, pp. 3594-3603, 1990.

[54] D. Javorsek II, D. Elmore, E. Fischbach et al., "New experimental limits on strongly interacting massive particles at the TeV scale," Physical Review Letters, vol. 87, no. 23, Article ID 231804, 4 pages, 2001.

[55] S. Mitra, "Uranus's anomalously low excess heat constrains strongly interacting dark matter," Physical Review D, vol. 70, no. 10, Article ID 103517, 6 pages, 2004.

[56] G. D. Mack, J. F. Beacom, and G. Bertone, "Towards closing the window on strongly interacting dark matter: far-reaching constraints from Earth's heat flow," Physical Review D, vol. 76, no. 4, Article ID 043523, 2007.

[57] D. Abrams, D. S. Akerib, M. S. Armel-Funkhouser et al., "Exclusion limits on the WIMP-nucleon cross section from the Cryogenic Dark Matter Search,” Physical Review D, vol. 66, Article ID 122003, 2002.

[58] D. S. Akerib, M. S. Armel-Funkhouser, M. J. Attisha et al., "Exclusion limits on the WIMP-nucleon cross section from the first run of the Cryogenic Dark Matter Search in the Soudan Underground Laboratory," Physical Review D, vol. 72, Article ID $052009,2005$. 
[59] Z. Ahmed, D. S. Akerib, S. Arrenberg et al., "Search for weakly interacting massive particles with the first five-tower data from the cryogenic dark matter search at the Soudan underground laboratory," Physical Review Letters, vol. 102, Article ID 011301, 2009.

[60] E. Aprile, K. Arisaka, F. Arneodo et al., "First dark matter results from the XENON100 experiment," Physical Review Letters, vol. 105, Article ID 131302, 2010.

[61] D. S. Akerib, H. M. Araujo, X. Bai et al., "First results from the LUX dark matter experiment at the Sanford Underground Research Facility," Physical Review Letters, vol. 112, Article ID 091303, 2014.

[62] R. Bernabei, P. Belli, R. Cerulli et al., "Search for WIMP annual modulation signature: results from DAMA/NaI-3 and DAMA/NaI-4 and the global combined analysis," Physics Letters $B$, vol. 480, pp. 23-31, 2000.

[63] R. Bernabei, P. Belli, F. Cappella et al., "Dark Matter search," Rivista del Nuovo Cimento, vol. 26, no. 1, 2003.

[64] R. Bernabei, P. Belli, F. Cappella et al., "First results from DAMA/LIBRA and the combined results with DAMA/NaI," European Physical Journal C, vol. 56, no. 3, pp. 333-355, 2008.

[65] G. Angloher, M. Bauer, I. Bavykina et al., "Results from 730 kg days of the CRESST-II Dark Matter Search," The European Physical Journal C, vol. 72, p. 1971, 2012.

[66] C. E. Aalseth, P. S. Barbeau, J. Colaresi et al., "Search for an annual modulation in a $p$-type point contact germanium dark matter detector," Physical Review Letters, vol. 107, Article ID 141301, 2011.

[67] M. Taoso, G. Bertone, and A. Masiero, "Dark matter candidates: a ten-point test," Journal of Cosmology and Astroparticle Physics, vol. 2008, no. 3, article 022, 2008.

[68] K. Kohri and T. Takahashi, "Cosmology with long-lived charged massive particles," Physics Letters B, vol. 682, pp. 337-341, 2010.

[69] M. Pospelov and J. Pradler, "Big bang nucleosynthesis as a probe of new physics," Annual Review of Nuclear and Particle Science, vol. 60, pp. 539-568, 2010.

[70] F. Sannino, "Conformal dynamics for TeV physics and cosmology," Acta Physica Polonica B, vol. 40, pp. 3533-3745, 2009.

[71] D. D. Dietrich and C. Kouvaris, "Constraining vectors and axial-vectors in walking technicolor by a holographic principle," Physical Review D, vol. 78, Article ID 055005, 2008.

[72] D. D. Dietrich and C. Kouvaris, "Generalized bottom-up holography and walking technicolor," Physical Review D, vol. 79, Article ID 075004, 2009.

[73] D. D. Dietrich, M. Jarvinen, and C. Kouvaris, "Linear confinement without dilaton in bottom-up holography for walking technicolour," Journal of High Energy Physics, vol. 2010, p. 23, 2010.

[74] R. Foadi, M. T. Frandsen, T. A. Ryttov, and F. Sannino, "Minimal walking technicolor: setup for collider physics," Physical Review D, vol. 76, no. 5, Article ID 055005, 2007.

[75] T. A. Ryttov and F. Sannino, "Ultraminimal technicolor and its dark matter technicolor interacting massive particles," Physical Review D, vol. 78, Article ID 115010, 2008.

[76] M. T. Frandsen and F. Sannino, "iTIMP: isotriplet technicolor interacting massive particle as dark matter," Physical Review D, vol. 81, Article ID 097704, 2010.

[77] C. Kouvaris, "Dark Majorana particles from the minimal walking technicolor theory," Physical Review D, vol. 76, Article ID 015011, 2007.
[78] K. Kainulainen, K. Tuominen, and J. Virkajarvi, "Weakly interacting dark matter particle of a minimal technicolor theory," Physical Review D, vol. 75, Article ID 085003, 2007.

[79] C. Kouvaris, "Dark side of strongly coupled theories," Physical Review D, vol. 78, Article ID 075024, 2008.

[80] K. Belotsky, M. Khlopov, and C. Kouvaris, "Muon flux limits for Majorana dark matter from strong coupling theories," Physical Review D, vol. 79, Article ID 083520, 2009.

[81] O. Antipin, M. Heikinheimo, and K. Tuominen, "Natural fourth generation of leptons," Journal of High Energy Physics, vol. 2009, p. 018, 2009.

[82] T. Hapola, M. Jarvinen, C. Kouvaris, P. Panci, and J. Virkajarvi, "Constraints on Majorana dark matter from a fourth lepton family," Journal of Cosmology and Astroparticle Physics, vol. 1402, p. 050, 2014.

[83] E. Nardi, F. Sannino, and A. Strumia, "Decaying dark matter can explain the $e^{ \pm}$excesses," Journal of Cosmology and Astroparticle Physics, vol. 2009, p. 043, 2009.

[84] A. Arvanitaki, S. Dimopoulos, S. Dubovsky, P. W. Graham, R. Harnik, and S. Rajendran, "Astrophysical probes of unification," Physical Review D, vol. 79, no. 10, Article ID 105022, 2009.

[85] M. W. Goodman and E. Witten, "Detectability of certain darkmatter candidates," Physical Review D, vol. 31, no. 12, pp. 30593063, 1985.

[86] PAMELA collaboration.

[87] M. Aguilar, G. Alberti, B. Alpat et al., "First result from the alpha magnetic spectrometer on the international space station: precision measurement of the positron fraction in primary cosmic rays of $0.5-350 \mathrm{GeV}$," Physical Review Letters, vol. 110, Article ID 141102, 2013.

[88] M. Ackermann, A. Albert, B. Anderson et al., "Dark matter constraints from observations of 25 Milky Way satellite galaxies with the fermi large area telescope," Physical Review D, vol. 89, Article ID 042001, 2014.

[89] M. Ackermann, M. Ajello, W. B. Atwood et al., "Constraints on the galactic halo dark matter from Fermi-LAT diffuse measurements," The Astrophysical Journal, vol. 761, p. 91, 2012.

[90] J. Buckley, D. F. Cowen, S. Profumo et al., "Cosmic Frontier indirect dark matter detection working group summary," http://arxiv.org/abs/1310.7040.

[91] A. Ibarra, D. Tran, and C. Weniger, "Indirect searches for decaying dark matter," International Journal of Modern Physics A, vol. 28, no. 27, Article ID 1330040, 2013.

[92] M. Tavakoli, I. Cholis, C. Evoli, and P. Ullio, "Constraints on dark matter annihilations from diffuse gamma-ray emission in the Galaxy," Journal of Cosmology and Astroparticle Physics, vol. 2014, p. 017, 2014.

[93] J. Conrad, "Indirect detection of dark matter with gammarays-status and perspectives," p. 048.

[94] L. Dugger, T. E. Jeltema, and S. Profumo, "Constraints on decaying dark matter from Fermi observations of nearby galaxies and clusters," Journal of Cosmology and Astroparticle Physics, vol. 2010, no. 12, article 015, 2010.

[95] A. Ibarra, A. S. Lamperstorfer, and J. Silk, "Dark matter annihilations and decays after the AMS-02 positron measurements," http://arxiv.org/abs/1309.2570.

[96] A. Strong and I. Moskalenko, "Propagation of cosmic-ray nucleons in the Galaxy," The Astrophysical Journal, vol. 509, no. 1, p. 212, 1998.

[97] T. Sjöstrand, S. Mrenna, and P. Skands, "PYTHIA 6.4 physics and manual," Journal of High Energy Physics, vol. 0605, p. 026, 2006. 
[98] L. J. Gleeson and W. I. Axford, "Solar modulation of galactic cosmic rays," Astrophysical Journal, vol. 154, p. 1011, 1968.

[99] E. A. Baltz and J. Edsjo, "Positron propagation and fluxes from neutralino annihilation in the halo," Physical Review D, vol. 59, no. 2, Article ID 023511, 1998.

[100] M. Cirelli and P. Panci, "Inverse Compton constraints on the Dark Matter $e^{ \pm}$excesses," Nuclear Physics B, vol. 821, no. 1-2, pp. 399-416, 2009.

[101] M. Ackermann, M. Ajello, A. Albert et al., "Fermi LAT search for dark matter in gamma-ray lines and the inclusive photon spectrum," Physical Review D, vol. 86, Article ID 022002, 2012. 

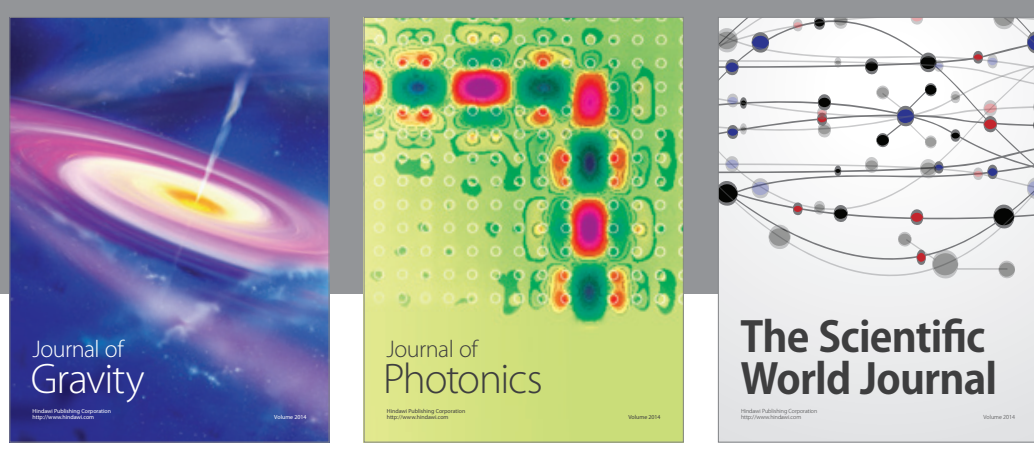

The Scientific World Journal
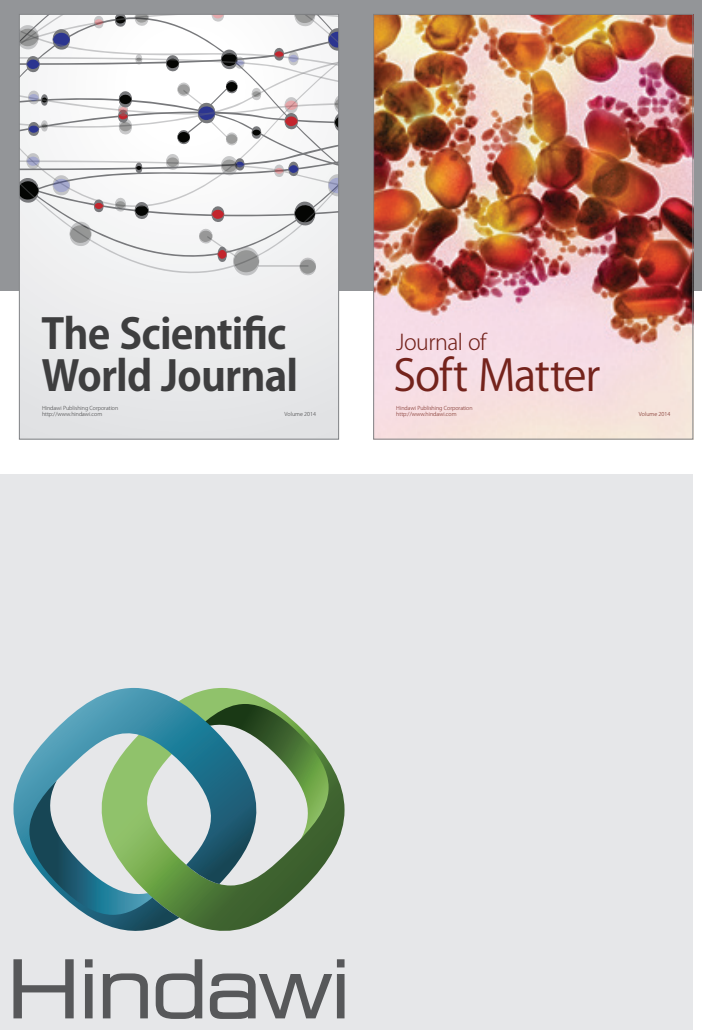

Submit your manuscripts at

http://www.hindawi.com

nternational Journal of

Statistical Mechanics
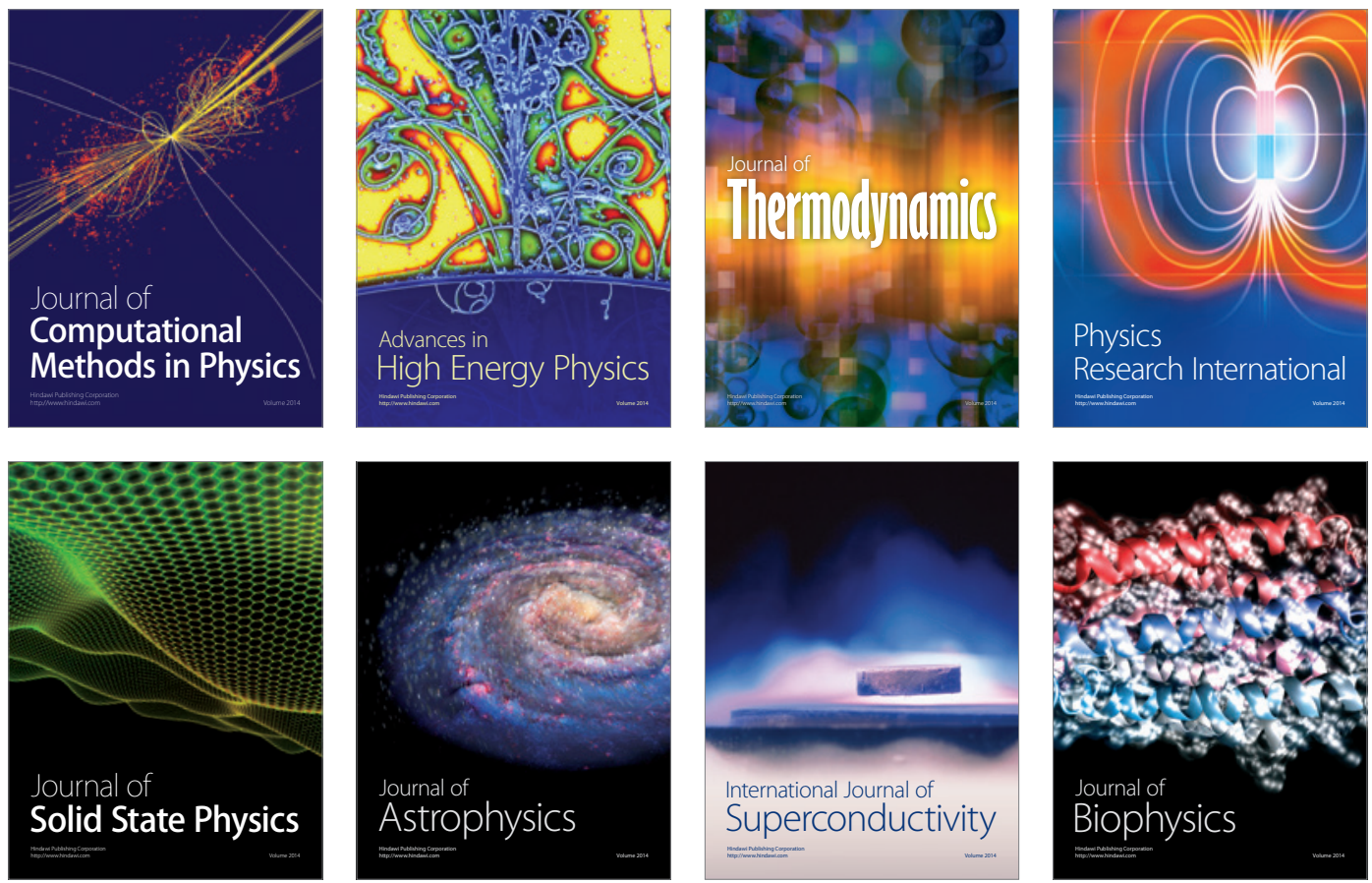
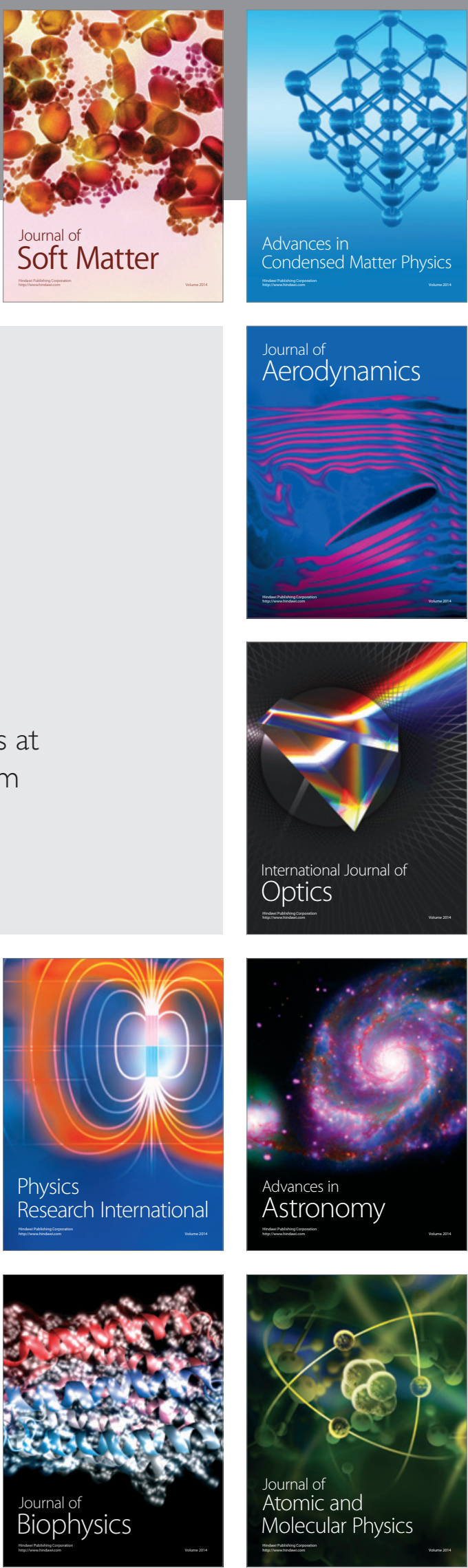